\title{
Análise e Recomendação de trajetos de aprendizagem em mundos virtuais
}

Felipe Becker Nunes, PPGIE, UFRGS, nunesfb@gmail.com

Manuel Constantino Zunguze, PPGIE/UFRGS - UPM, manuelczunguze@gmail.com

Fabrício Herpich, PPGIE, UFRGS, fabricio.herpich@gmail.com

Aline Grunewald Nichele, aline.nichele@poa.ifrs.edu.br

Liane Margarida Rockenbach Tarouco, PPGIE, UFRGS, liane@penta.ufrgs.br

José Valdeni de Lima, PPGIE, UFRGS, valdeni@inf.ufrgs.br

Resumo. Non-Player Characters são entidades programáveis que executam atividades pré-definidas. Eles podem ser utilizados nos mundos virtuais no papel de tutores que auxiliam os alunos durante atividades educacionais, tendo como base informações coletadas durante as interações destes. Este artigo apresenta uma proposta de uso de agentes NPC em mundos virtuais para prover sugestões de trajetórias de aprendizagem com base nas interações dos indivíduos. Foi utilizado o ambiente OpenSim onde, através de scripts de monitoramento das atividades foram formuladas sugestões de trajetórias. Como resultados, através do feedback positivo dos participantes constatouse que os agentes NPC propuseram de forma eficaz trajetórias de aprendizagem.

Palavras - Chave: agentes, mundos virtuais, trajetórias de aprendizagem.

\section{Tracking and Assessment of Student's in Virtual Worlds}

\begin{abstract}
NPC agents can be used in virtual worlds in the role of tutors, who assist students during educational activities, based on information collected during their interactions. This article presents a proposal to use NPC agents in virtual worlds to provide suggestions for learning trajectories based on the interactions of individuals. OpenSim environment was used, using scripts to monitor the students and formulate the suggestions by the agents. As results, it was verified the feasibility of this proposal with the correct functioning of the resources in the tests performed and positive feedback from the participants.
\end{abstract}

Keywords: agents, virtual worlds, learning trajectories.

\section{INTRODUÇÃO}

O crescente uso das tecnologias de informação e comunicação no contexto educacional tem possibilitado a introdução e adaptação de metodologias de ensino e o uso de ambientes digitais de aprendizagem. Os mundos virtuais surgem como alternativa para auxiliar no processo de aprendizagem, principalmente possibilitando a imersão, aprendizagem ativa, colaboração e comunicação, assim como fornecendo recursos de simulação de experimentos provenientes deste ambiente.

Dalgarno \& Lee (2010) veem os mundos virtuais como um espaço onde os alunos podem criar e manipular objetos digitais, explorar novas formas de interação, incorporar experiências e interagir com os outros usuários através de um avatar (representação virtual do usuário). Estes ambientes permitem também a criação de agentes Non-Player Characters (NPC), que são avatares controlados por scripts de programação que podem realizar ações pré-determinadas ou dinâmicas, conforme seu modo de configuração.

No âmbito educacional, os NPC podem desempenhar um papel semelhante ao de um tutor, por meio de uma ligação com uma base de conhecimento de um chatterbot. Este tipo de entidade são programas de computador que tentam simular conversações 
com os usuários, com objetivo de pelo menos temporariamente, levar um ser humano a pensar que está conversando com outra pessoa (PILASTRI e BREGA, 2009).

Atualmente, devido aos custos elevados e falta de infraestrutura para a contratação em grande escala de tutores, tal ação de tutoria poderia vir a ser suprida, pelo menos em parte, dentro do mundo virtual por agentes NPC interconectados a um chatterbot. Desta forma, os alunos podem interagir no mundo virtual com recursos como slides, vídeos e simulações, sendo auxiliados por agentes NPC, que poderiam tirar dúvidas sobre as atividades, instigar a busca por respostas, fornecer dicas e questionar os usuários.

A interação dos alunos com os recursos do mundo virtual e com os agentes NPC possibilita a coleta de diversos tipos de dados, conforme pode ser visto no trabalho de Nunes et al. (2016) em que o presente artigo faz parte do projeto iniciado neste estudo. Os dados armazenados abrem a possibilidade para que seja explorado e mapeado os trajetos percorridos pelos usuários durante cada interação no ambiente.

Saint-Georges e Filliettaz (2008) entendem que este tipo de mapeamento permite inferir o trajeto efetivamente realizado por cada estudante e a possibilidade de monitoramento com vistas a um planejamento de longo prazo por parte do professor. Os autores explicam que o uso deste termo está ligado a duas proposições: a perspectiva situacional e a ideia de trajetória. A primeira diz respeito ao foco nas ações que ocorrem em tempo real e são realizados pelos indivíduos, enquanto a segunda envolve a ideia de trajetória, que implica em ter uma visão mais futurista, levando em consideração ações à longo prazo.

Portanto, tanto na parte de monitoramento do aluno (para verificar quais ações ele tem desempenhado nas interações no mundo virtual), quanto no planejamento das atividades pelo professor, a análise das trajetórias dos alunos poderia ser aplicada. Desta forma, este artigo apresenta uma proposta utilizando um mundo virtual criado no OpenSim, em que agentes NPC interconectados a um chatterbot analisam os dados coletados das interações dos usuários. Estes agentes buscam formular e apresentar sugestões de trajetos baseados nos materiais de aprendizagem presentes no ambiente. Tal proposição visa auxiliar aos alunos durante sua interação propondo um percurso pedagógico para ser percorrido no ambiente, assim como também armazenar os dados provenientes para futuras análises relacionadas à avaliação do aluno e ao planejamento por parte do professor.

\section{TRABALHOS RELACIONADOS}

Com o objetivo de situar o leitor acerca de algumas pesquisas já desenvolvidas envolvendo o escopo deste trabalho, e, esclarecer as diferenças existentes nesta proposta, são descritas algumas observações. No que concerne ao campo da Computação Afetiva, iniciativas têm sido desenvolvidas com o uso de agentes NPC e a atribuição de emoções nas ações e papéis desempenhados por este tipo de avatares nos Mundos Virtuais.

Um dos principais aspectos que tem sido abordado está relacionado à motivação e influência que estes tipos de agentes podem promover na área educacional, como pode ser visto no trabalho de Soliman e Guetl (2014). No trabalho de Shiban et al. (2015), a análise efetuada esteve direcionada no impacto que a aparência de agentes virtuais tem no papel de tutores durante um curso a distância, tendo como resultados positivos que este aspecto pode ser visto como um fator de impacto na interação dos alunos.

$\mathrm{Na}$ área de computação sensível do contexto, também tem sido desenvolvidos trabalhos relacionados à percepção do agente em captar determinadas informações dos 
usuários que auxiliem na adaptação do tipo de ação e conceito que será emitido a ele. Como exemplo, na pesquisa realizada por Herpich (2015), agentes NPC identificam a expertise do usuário em relação a um determinado tópico e adaptam o tipo de conceito e explicação que será fornecida a ele com base nestas informações.

É possível encontrar em comum nesta pesquisa a necessidade de coleta de dados resultantes das interações dos usuários no mundo virtual para a tomada de decisão do agente, assim como aspectos relacionados à forma de interação e aparência deste também devem ser considerados. A diferença presente nesta pesquisa está na proposição de uma análise dos dados para a formulação de sugestões de trajetórias de aprendizagem que podem ser seguidas pelos alunos e utilizadas pelo professor em seu planejamento.

\section{MUNDOS VIRTUAIS A AGENTES NPC}

Os mundos virtuais são ambientes que simulam diferentes tipos de objetos e interações do mundo real de forma gráfica tridimensional, podendo ser aplicados com um enfoque educacional. Rafalski et al. (2014) explicam que os alunos deixam de ser meros espectadores dos acontecimentos, outrora exclusivos à sala de aula, para serem protagonistas do processo de aprendizagem nestes ambientes de autoria e cooperação.

Dentre os ambientes disponíveis, pode-se citar o Second Life, Open Wonderland e o OpenSim, tendo sido selecionado o OpenSim para ser utilizado nesta pesquisa. Para visualizar o ambiente, os participantes necessitam instalar um viewer em sua máquina para acessar com suas credenciais, o servidor que hospeda o mundo virtual. Exemplos de viewers são o Singularity, Firestorm e Imprudence.

Do ponto de vista educacional, Nunes et al. (2016) ressaltam a utilização de diversos recursos como forma de apoio ao processo de aprendizagem, no qual podem ser usados vídeos, slides, questionários e navegação em sites diretamente no ambiente. Com relação à construção de simulações interativas, o uso de scripts de programação na linguagem OSSL (OpenSim Script Language), nativa do ambiente, pode ser utilizado em consonância com os objetos 3D disponíveis no mundo virtual.

A programação de scripts OSSL também permite a construção e configuração de agentes NPC no mundo virtual, os quais podem ser interconectados a uma base de conhecimento de um chatterbot, que permitirá realizar a conversação com demais usuários no ambiente. A linguagem de especificação da base de conhecimento é a Artificial Intelligence Markup Language (AIML), que permite usar uma base de conhecimento já existente ou criar uma nova base (SGOBBI et al., 2014). Nesta pesquisa será utilizado o Program $O$, que se trata de um servidor on-line que permite a criação de um chatterbot com uma base de conhecimento pré-definida ou a ser adaptada, se necessário.

No momento que o agente conversacional NPC detém o conhecimento que está sendo debatido, o mesmo pode processar a requisição e expressar sua resposta de acordo com aquilo que sua pesquisa retornou. Porém, nas circunstâncias em que o chatterbot não dispõe de determinada informação em sua base de dados, o mesmo irá empenhar-se em conduzir a conversação até que as expressões utilizadas pelo estudante sejam encontradas na base de conhecimento do agente conversacional.

\section{TRAJETÓRIAS DE APRENDIZAGEM}

Júnior (2005) esclarece que o processo de análise do percurso pedagógico percorrido pelo aluno durante uma intervenção didática é uma prática encarada como a rota de aprendizagem que o aluno trilha, com o objetivo de atingir o conhecimento 
necessário acerca de um ou vários conceitos interligados a um tópico geral. Conforme descrito na introdução deste artigo, Saint-Georges e Filliettaz (2008) creditam este termo como sendo a definição situacional de uma trajetória, buscando monitorá-la com vistas a um planejamento de longo prazo.

Wilson et al. (2013) enfatiza que diferentes níveis de aprendizagem e particularidades são esperados, não devendo ser tratados como algo estático e equiparado a um único caminho correto a ser cumprido. É importante ressaltar que as rotas percorridas pelos alunos não são padronizadas, o que acarreta na interpretação de que cada indivíduo percorre um caminho pessoal e distinto ao longo de uma atividade.

Simon (1995) explica que o docente está constantemente percebendo a extensão das modificações e transformações que podem ser construídas por algum ou todos os componentes da trajetória hipotética de aprendizagem: o método, as atividades e o processamento hipotético da aprendizagem. O conhecimento que o professor detém de cada indivíduo que compõe o grupo de alunos sob sua orientação, poderá fornecer o embasamento necessário para o entendimento do caminho genérico a ser proposto pelo docente, assim como os motivos pelos quais determinado caminho foi percorrido pelos alunos durante a realização de um conjunto de atividades didáticas.

No escopo deste trabalho, o processo de apresentação de possíveis trajetórias de aprendizagem a serem seguidas pelos alunos e planejadas pelo docente, está centralizada na coleta de dados das interações no mundo virtual, com sua análise e processamento automatizado. Torna-se necessário compreender que o mapeamento deste tipo de trajetórias deve ser considerado como um processo contínuo, não o tornando um julgamento dicotômico entre certo e errado.

\section{PROCEDIMENTOS METODOLÓGICOS}

A pesquisa teve natureza explicativa, abordagem quali-quantitativo e modalidade do tipo quase-experimental. A primeira etapa adotada foi a realização de uma revisão de literatura acerca dos tópicos relacionados a mundos virtuais, agentes NPC e trajetórias de aprendizagem. Assim como, também foi realizada uma pesquisa acerca dos trabalhos relacionados na área de agentes NPC. A infraestrutura tecnológica necessária para a realização deste experimento foi definida, sendo selecionado o mundo virtual OpenSim, que se trata de uma aplicação open source. O visualizador adotado foi o Singularity, por ser gratuito, adequado às necessidades de visualização e criação de objetos no ambiente.

Foi construído um laboratório para o ensino de ciências no mundo virtual contendo seis tipos de salas com materiais diferentes: vídeos, slides, textos, questões, imagens e simulações. O conteúdo programático abordado foi molécula da água, pressão, tipos de água, ciclo da água e tratamento de água e esgoto. Em cada sala foram inseridos sensores programados com scripts que coletavam informações referentes às interações dos usuários (Nunes et al., 2016), sendo melhor detalhado na próxima seção. Também foram inseridos diversos agentes NPC no laboratório para auxiliar os alunos e fornecer sugestões de trajetórias de aprendizagem a serem seguidas, todos executavam as mesmas funções e tinha a base de conhecimento igual.

Para a validação da proposta apresentada, foram selecionados 11 professores em formação do curso de Licenciatura em Ciências da Natureza: Habilitação em Biologia e Química de uma universidade federal. Os participantes tiveram 2 encontros presenciais em um laboratório de informática da instituição de ensino com duração de uma hora e quarenta e cinco minutos para cada um destes encontros. No primeiro encontro foi realizada a ambientação dos participantes ao mundo virtual, no qual eles aprenderam a 
utilizar os comandos básicos do ambiente OpenSim, personalizaram seus avatares e realizaram uma breve visita ao laboratório no mundo virtual.

No segundo encontro, os participantes interagiram com os recursos presentes no laboratório e com os agentes NPC dispostos em todo ambiente. Por fim, um questionário composto por nove questões de múltipla escolha com cinco opções de resposta seguindo a escala Likert (1932) e uma questão dissertativa foi aplicado para coletar informações referentes a opinião do usuário quanto ao uso do agente, assim como dificuldades e vantagens que foram identificados durante a interação com ele. A análise dos dados foi realizada utilizando a medição de porcentagem das respostas e a mediana de cada questão para verificar a opinião dos usuários em diferentes questões relacionadas ao uso do agente.

\section{PROTÓTIPO E RESULTADOS INICIAIS}

O objetivo do estudo de caso aplicado foi a utilização dos recursos no ambiente para a coleta de dados, de forma que o agente pudesse interagir com os participantes, fornecendo sugestões de trajetórias baseadas nestas interações ocorridas. Assim, os participantes poderiam ter uma experiência juntamente ao agente, no qual teriam condições de responder e avaliar sua atuação de forma consistente e integra.

Para a descrição do experimento realizado, se configura necessário realizar primeiramente o detalhamento da coleta dos dados no mundo virtual. Tal processo se inicia com a execução de scripts no ambiente, que monitoram constantemente a presença dos usuários. Conforme já explicitado, este trabalho é parte integrante do estudo iniciado em Nunes et al. (2016), assim, os scripts utilizados neste estudo são baseados no trabalho realizado anteriormente.

No escopo desta pesquisa, foi estabelecida a criação de seis salas em um laboratório virtual, cada uma com diferentes tipos de materiais de ensino: vídeos, slides, textos, questões, imagens e simulações. Scripts que monitoram a presença do usuário foram inseridos em cada uma destas salas, sendo armazenados os registros em um banco de dados. Os dados armazenados foram os seguintes: nome do usuário, nome do laboratório, nome da sala, seu comportamento (parado, andando, voando, escrevendo e ausente), o tempo de permanência na sala, a data e a hora do registro. Tais dados irão ajudar o agente NPC a formular sugestões acerca dos tipos de materiais didáticos que podem ser de preferência do aluno em uma sequência de trajetória.

O usuário ao entrar no laboratório pode clicar no painel disponibilizado para solicitar ajuda ao agente NPC, que lhe dá as boas-vindas e explica que irá ajudá-lo nas atividades em cada sala do laboratório. Efetuado os esclarecimentos iniciais necessários para contextualizar o aluno, o agente NPC realiza uma busca na base de dados para verificar quais os tipos de materiais que têm sido mais visualizados pelo aluno. Caso seja seu primeiro acesso, o aluno não terá dados ainda, portanto o agente NPC o questiona sobre suas salas preferenciais no laboratório, afim de obter dados que, embora possam ser menos precisos, irão ajudar nas etapas posteriores do processo.

Formuladas as sugestões, o agente NPC apresenta as considerações gerais para o aluno, explicando seu funcionamento e os tópicos que estão sendo abordados no laboratório. Foi decidido por manter o agente parado, sem seguir o usuário, de forma que quando ele tenha dúvidas, se dirija ao agente e para que ele não fique todo o tempo seguindo o participante e evitando uma monitoria demasiadamente guiada. Efetuadas estas ações, o agente NPC grava a sugestão que ele deu ao aluno para posteriores consultas. Executadas todas ações programadas, o agente NPC permanece à disposição do usuário para tirar dúvidas. 
No que concerne a interação do usuário com o agente a partir do seu segundo acesso, quando entrar no ambiente o usuário solicita ajuda ao agente NPC, que lhe dá as boas-vindas e realiza uma busca na base de dados para verificar as sugestões sobre conteúdos que foram fornecidas. Neste momento, ele questiona o aluno se aprovou ou não a última sugestão que tinha sido comunicada a ele, armazenando na base de dados a resposta fornecida pelo aluno.

Posteriormente, ele processa os dados contidos sobre o número de visitas e o tempo que permaneceu em cada uma das salas do laboratório, levando em conta a média de cada atributo para verificar os locais que ele possui maior preferência. Desta forma, o agente NPC irá processar estes dados e verificar qual o local mais vezes foi utilizado pelo aluno, formulando a sugestão com base nestas informações e nas processadas anteriormente, com o objetivo de comunicar ao aluno sua recomendação.

Com base nisso, o agente NPC formula duas sugestões de trajetórias de aprendizagem aos alunos:

- A primeira é uma trajetória de aprendizagem sequencial, mostrando as seis salas presentes no laboratório em ordem crescente de maior tempo e número de visitas com base nas interações do usuário.

- A segunda sugestão é uma trajetória de aprendizagem criada pelo professor com base em sua expertise, contendo as seis salas do laboratório na sequência que ele considera mais adequada para o conteúdo abordado.

Um exemplo de trajetória é apresentado na Figura 1. Desta forma, o aluno possui a sua disposição duas opções de trajetórias de aprendizagem para serem seguidas durante sua interação no ambiente, ressaltando que as trajetórias são dinâmicas, podendo ser mudadas pelo professor e também a do aluno, que é formulada a cada solicitação realizada ao agente. Portanto, se o aluno interagir no ambiente e solicitar sua trajetória, ela poderá ser diferente da anterior, visto que é calculada em tempo real.

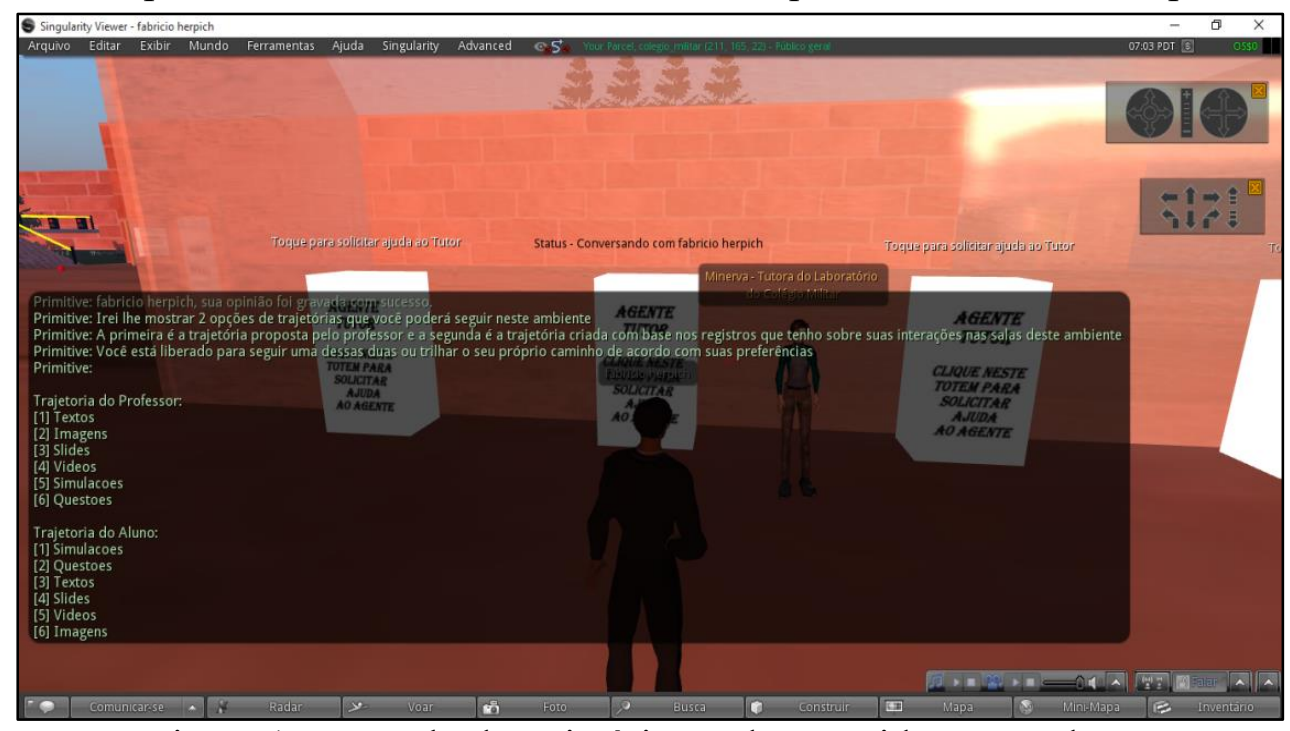

Figura 1. Exemplo de trajetória sendo sugerida ao estudante.

É importante ressaltar que o aluno não é obrigado a seguir as duas sugestões de trajetórias de aprendizagem apresentadas a ele, podendo seguir seu próprio percurso pedagógico. Foi criada uma segunda tabela no banco de dados para gravar as sugestões apresentadas pelo agente ao estudante, sendo armazenados os seguintes dados: Identificador, nome do usuário e laboratório que está interagindo; O status para saber se gostou da última sugestão fornecida; A trajetória fornecida ao estudante com base em 
suas interações; A trajetória fornecida pelo professor; A trajetória que foi seguida pelo aluno no mundo virtual; Data e hora do registro;

Após ter desempenhado estas ações, o agente fica à disposição do usuário para tirar dúvidas que possam surgir. Visto que a base de conhecimento do Program O pode ser manipulada e alterada, tornou-se possível inserir informações acerca dos conteúdos específicos trabalhados no mundo virtual. Assim, o agente NPC tem condições de responder questionamentos específicos do conteúdo que o usuário está estudando no laboratório.

É importante ressaltar que para cada usuário é criado um agente NPC no mundo virtual quando este entra no laboratório, sendo esta conversação exclusiva entre o aluno e o agente que foi designado para ele. Visto que as informações que são compartilhadas por meio do uso do chat podem se misturar, assim como o processo como um todo, foi optado por individualizar o atendimento de cada usuário, buscando fornecer uma tutoria exclusiva.

A trajetória que o aluno seguir no ambiente é armazenada justamente para fins de comparação e análise, podendo assim ser verificado se o aluno seguiu alguma das duas sugestões fornecidas ou seguiu um caminho alternativo a elas. Também é armazenada a sua opinião quanto a sugestão para saber seu feedback a este processo.

Um aspecto importante do ponto de vista docente é que este tipo de dado pode ser consultado pelo professor para analisar como está sendo a interação de cada aluno no mundo virtual, assim como pode ser analisado todos os caminhos para visualizar a trajetória mais adotada por uma turma ou grupo de alunos. A seção a seguir apresenta os resultados obtidos com a validação desta proposta.

\subsection{Resultados Preliminares}

Para validar o protótipo apresentado, foram realizados testes com 11 usuários, conforme descrito anteriormente nos procedimentos metodológicos deste trabalho. A análise e discussão das respostas para as nove questões objetivas são descritas a seguir, podendo ser visualizada na Figura 2 uma visão geral das porcentagens das respostas dos usuários. Com base nas respostas da questão dissertativa e observações realizadas durante o experimento, também foi possível realizar uma reflexão em cada questão objetiva.

A questão 1 buscou indagar os usuários acerca das sugestões iniciais fornecidas pelo agente NPC para entender seu modo de funcionamento, e também, esclarecer ao usuário detalhes referentes às salas existentes no laboratório. Mais de $70 \%$ dos participantes assinalaram de forma positiva que o agente conseguiu esclarecer estes detalhes. Tal asserção está interligada a segunda questão que aborda a facilidade de navegação no ambiente com as explicações do agente NPC, em que também mais de $70 \%$ dos usuários assinalaram de forma positiva acerca disso.

Estes indícios de satisfação demonstram que a rotina inserida no agente pode ser considerada como uma alternativa válida, ressaltando que as críticas estão focadas no agente ainda ser considerado muito "mecânico". Isso se deve ao fato de sua conversação inicial estar ainda estática, com o mesmo texto repetidamente e sem mudanças nesta parte inicial, além de não possuir movimentos gestuais que possam deixar sua interação mais realística. Zhou et al. (2006) explicam que a emoção em um NPC vai aumentar sua credibilidade, visto que a expressividade de emoções com hábitos especiais faz o NPC se tornar mais distinto em personalidades.

A questão 3 estava relacionada à base de conhecimento adaptada ao conteúdo abordado no experimento, em que houve uma divisão um pouco maior nas respostas fornecidas, sendo que em torno de $70 \%$ dos usuários concordaram que o agente 
conseguiu responder de forma satisfatória aos questionamentos. Demais críticas estão centralizadas no fato que o agente somente responde por meio de palavras-chave, sendo que segundo os participantes, se não fosse a tabela de instruções, ficaria mais difícil de conversar com ele e tirar dúvidas, realizando perguntas mais gerais com os termos chave misturados.

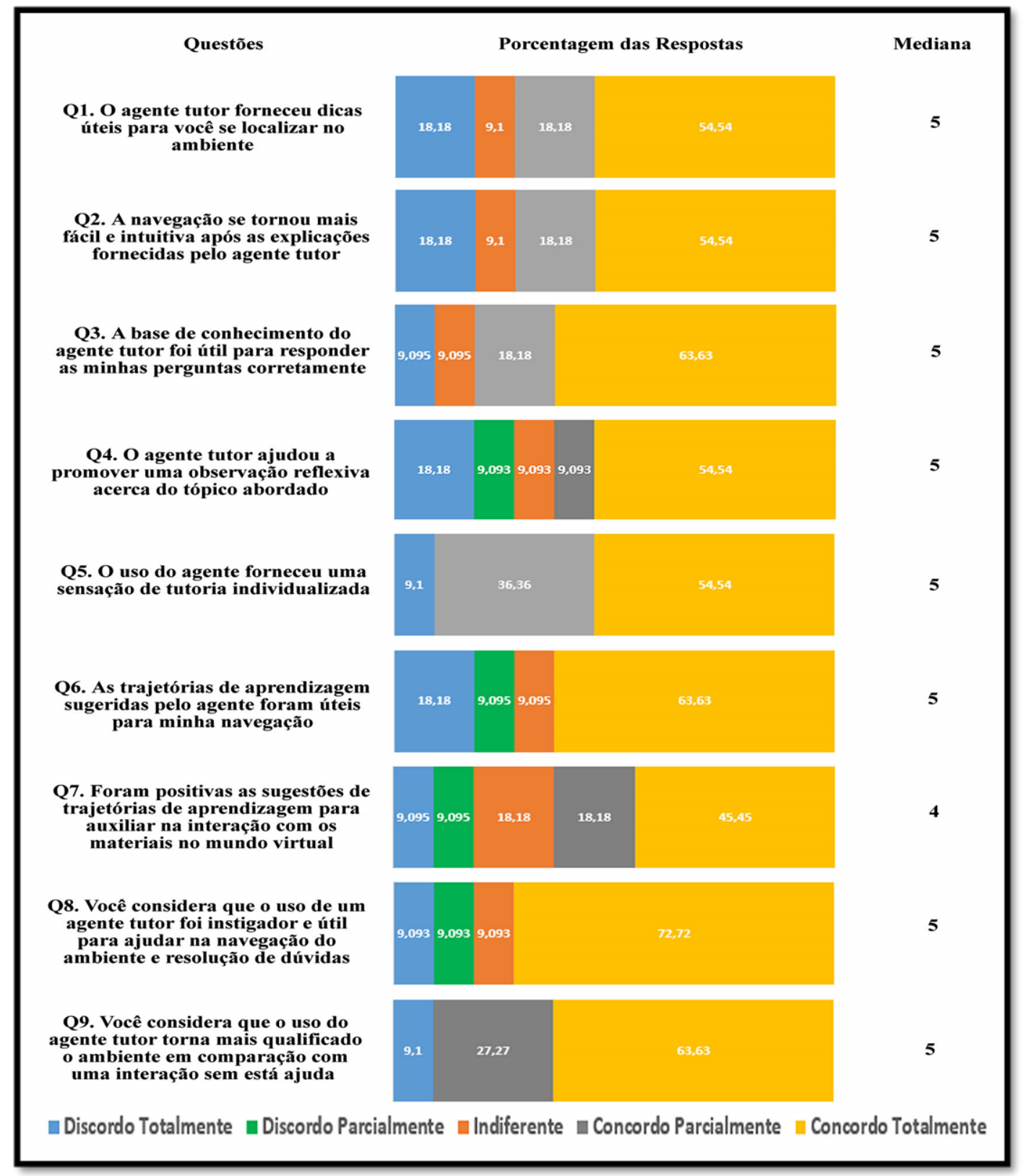

Figura 2. Resultados de cada questão avaliativa do experimento

Tais fatores impactaram nas respostas da questão 4, na qual foi questionado acerca da reflexão do usuário a partir das respostas fornecidas sobre os conteúdos abordados no experimento. Em torno de 63,5 \% concordaram com tal asserção, o que pode ser considerado positivo, sendo a principal crítica relacionada à facilidade de conversa com o tutor, em que os participantes consideraram que deveria ser possível trocar ideias mais elaboradas com ele para haver uma conversa mais reflexiva sobre o assunto abordado. Sgobbi et al. (2014) ressaltam dentro deste contexto que o agente precisa proporcionar aos usuários orientação e apoio, para que estes possam realizar as atividades previstas. 
No que concerne a questão 5 , pode se considerar muito positivo o feedback obtido pelos usuários, visto que mais de $90 \%$ deles concordou que a presença do tutor reforçou a sensação de se ter um tutor individualizado. Tal fato resulta de o agente ter sido programado para não conversar com os demais usuários enquanto fornece ajuda a um participante, justamente com o objetivo de reforçar o papel de tutor individualizado. A questão 6 abordou diretamente a opinião dos participantes sobre as trajetórias de aprendizagem apresentadas pelo agente durante a interação, sendo que em torno de $63 \%$ dos usuários concordaram com esta afirmação. Demais valores negativos estão ligados aos comentários feitos pelos alunos, em que eles reportaram dificuldades sobre o entendimento deste recurso e como ele funcionava.

A questão 7 também está interligada a isto, sendo questionada a validade e utilidade das trajetórias apresentadas, resultando também em aproximadamente $63 \%$ de aceitação. Isso pode ser considerado natural, visto que somente dois encontros foram realizados com poucas interações, sendo um ambiente com uma proposta diferente da que eles estavam utilizando normalmente (Ambiente Moodle), o que acaba por vezes sendo uma carga elevada de novas informações, tornando mais difícil de ser assimilada pelos participantes em pouco tempo, sendo necessário um maior período de tempo.

Na questão 8 foi solicitada a opinião acerca da utilização do agente, se ele conseguiu instigar e ajudar o usuário em sua interação com o ambiente e com $o$ conteúdo abordado, sendo que em torno de $72 \%$ deles concordaram com tal asserção. Este resultado predominantemente positivo pode ser considerado motivador, visto que um dos principais objetivos dos agentes NPC inseridos no ambiente estava centralizado neste ponto. Concomitantemente a este ponto está a questão 9, em que os participantes responderam se a presença dos agentes NPC no ambiente tornava mais qualificada a interação em comparação com um ambiente no qual ele não teria esta ajuda, tendo um retorno demasiadamente positivo com mais de $90 \%$ de concordância. Isso demonstra a validade da presença e apoio fornecido por esta entidade, havendo de forma geral necessidade de aprimorar seus comportamentos e raciocínios.

Por fim, demais pontos destacados nos comentários fornecidos pelos participantes estiveram centralizados na maior caracterização realística do agente, em que ele deveria estar vestido com roupas adequadas para o laboratório, assim como também foi sugerido que ele deveria acompanhar o aluno durante seu trajeto no ambiente. Também foi reconhecido por diversos participantes que o agente está em fase inicial, sendo estas sugestões fornecidas para aprimorar suas habilidades.

\section{CONCLUSÃO}

A utilização dos mundos virtuais e agentes NPC ganhou força no decorrer dos últimos anos no campo educacional, sendo desenvolvidas diversas iniciativas com o objetivo de possibilitar a integração destes recursos com as atividades educacionais realizadas pelos alunos em diferentes áreas de atuação. A proposta deste artigo envolvendo a inserção de agentes NPC em laboratórios virtuais para fornecerem ajuda durante as interações e também proporem sugestões de trajetórias de aprendizagem de forma dinâmica, pôde ser considerada instigadora e válida com base nos resultados obtidos neste experimento.

As impressões iniciais deste estudo foram positivas e demonstram que a proposta é viável e pode ser colocada em prática de forma mais consistente e duradoura. Ressalta-se a importância de considerar todas as críticas construtivas apresentadas pelos participantes e reconhecer que o agente está situado em um estágio inicial, sendo necessário evoluir seus recursos para atender às demandas solicitadas. $\mathrm{O}$ trabalho 
apresentado neste artigo não abrange o impacto desta abordagem na aprendizagem dos alunos, mas a viabilidade da proposta e sua forma de aplicação.

O trabalho realizado neste projeto está dentro do contexto socializado por Wilson et al. (2013), em que a análise das trajetórias dos alunos permite identificar evidências que possam mostrar os seus diferentes estágios de progresso, o que possibilita tentar adaptar as instruções e conteúdos conforme as necessidades individuais para que os alunos atinjam o desempenho final desejado. Desta forma, o professor poderia considerar uma análise dos estilos de aprendizagem dos alunos e/ou novos métodos de aprendizagem para serem aplicados de acordo com as informações das trajetórias.

Como trabalhos futuros, está a realização das melhorias descritas na análise dos resultados deste experimento, assim como promover interações mais duradouras com usuários para viabilizar sua real contribuição do ponto de vista educacional. Isso se faz necessário visto que um tempo mais prolongado com usuários utilizando o ambiente e o agente com diferentes tópicos de ensino permitirá mensurar de forma qualitativa e/ou quantitativa sua real contribuição no processo de aprendizagem dos alunos.

\section{REFERÊNCIAS BIBLIOGRÁFICAS}

RAFALSKI, J. P., JÚNIOR, R. R. M. V., SILVA, C. A. S. Mundos Virtuais como Suporte à Aprendizagem - Uma Avaliação na Implementação de Projetos de Aprendizagem, Renote, v. 12, n. 1, p. 1-10, 2014.

DALGARNO, B. \& LEE, M. J.W. What are the learning affordances of 3-D virtual environments? British Journal of Educational Technology, v. 41, n. 1, p. 10-32, 2010.

HERPICH, F. "ELAI: Intelligent Agent adaptive to the Level of Expertise of students". Dissertação de Mestrado em Ciência da Computação, UFSM, Santa Maria, 2015.

JÚNIOR, G. D. C. Trajetórias de aprendizagem de alunos de ensino médio: produção de significados em um curso introdutório de física térmica, Dissertação de Mestrado, 2005.

LIKERT, R. A technique for the measurement of attitudes. Archives of Psychology, 1932.

NUNES, F. B., HERPICH, F., TAROUCO, L. M. R., DE LIMA, J. V. Monitoramento e Avaliação de Estudantes em Mundos Virtuais. Renote, v. 14, n. 1, p. 1-10, 2016.

PILASTRI, A. L.; BREGA, J. R. F. Chatterbot com Interatividade ao Avatar Encapsulado no Ambiente Virtual Second Life usando a base de conhecimento em AIML. Workshop de Realidade Virtual e Aumentada - Unisanta, 2009.

SAINT-GEORGES, I.; FILLIETTAZ, L. Situated trajectories of learning in vocational training interactions. European Journal of Psychology of Education, v. 23, n. 2, p. 213233, 2008.

SGOBBI, F. S.; NUNES, F. B.; BOS, A. S.; BERNARDI, G.; TAROUCO, L. M. R. Interação com artefatos e personagens artificiais em mundos virtuais. Simpósio Brasileiro de Informática na Educação, p. 1-10, 2014.

SHIBAN, Y.; SCHELHORN, I.; JOBST, V.; et al. The appearance effect: Influences of virtual agent features on performance and motivation. Computers in Human Behavior, v. 49, p. 5-11, 2015.

SIMON, M. A. Reconstructing mathematics pedagogy from a constructivist perspective. Journal for Research in Mathematics Education, v. 26, n. 2, p. 114-145, 1995.

SOLIMAN, M.; GUETL, C. Evaluation Study and Results of Intelligent Pedagogical Agent-led Learning Scenarios in a Virtual World. MIPRO, p .26-30, 2014.

WILSON, P. H.; MOJICA, G. F.; CONFREY, J. Learning trajectories in teacher education: Supporting teacher's understandings of student's mathematical thinking. Journal of Mathematical Behavior, v. 32, p. 103- 121, 2013.

ZHOU, C.; YU, X.; SUN, J.; YAN, X. Affective Computation Based NPC Behaviors Modeling. Proceedings of the IEEE/WIC/ACM, p. 1-4, 2006.

V. $15 \mathrm{~N}^{\mathrm{o}}$ 1, julho, 2017 